\title{
HIGH-PRECISION TIMING OF MILLISECOND PULSARS
}

\section{AND PRECISION ASTROMETRY}

\author{
V. M. KASPI \\ IPAC/Caltech/Jet Propulsion Laboratory \\ 770 So. Wilson Ave \\ Pasadena, CA 91125
}

\begin{abstract}
.
We present the technique of long-term, high-precision timing of millisecond pulsars as applied to precision astrometry. We provide a tutorial on pulsars and pulsar timing, as well as up-to-date results of long-term timing observations of two millisecond pulsars, PSRs B1855+09 and B1937+21. We consider the feasibility of tying the extragalactic and optical reference frames to that defined by solar system objects, and we conclude that precision astrometry from millisecond pulsar timing has a bright future.
\end{abstract}

\section{Introduction}

Pulsars are rapidly rotating, highly magnetized neutron stars that emit a beam of light that appears as a broad-band pulse of radiation once per rotation period. What is now referred to as the "slow" pulsar population includes over 600 pulsars with rotation periods in the range $33 \mathrm{~ms}<P<$ $5 \mathrm{~s}$. What is striking about most of these sources is that their rotation is extremely stable, owing to their large rotational inertia. Rotation periods with 12 significant digits are generally straightforward to measure in just a few years of observations, and are typically limited only by measurement 
uncertainties. The discovery of the first millisecond pulsar by Backer et al. (1982) made the timing of "slow" pulsars seem like child's play: the rotational period of the millisecond pulsar PSR B1937+21 has been measured with fractional uncertainty $10^{-15}$ (Kaspi, Taylor \& Ryba 1994).

It is this rotational stability, combined with the Earth's motion around the Sun, that allows high-precision astrometry to be accomplished through pulsar timing. In this paper, we review the technique of pulsar timing with an emphasis on astrometric applications. It must be noted, however, that astrometry is only one of many byproducts of timing observations. Other equally interesting and important fields addressed in high-precision timing include general relativity, cosmology, time-keeping metrology, interstellar medium physics, orbital evolution, as well as neutron star physics.

\section{Fundamentals of Pulsar Timing}

\subsection{TIMING OBSERVATIONS}

Although a handful of pulsars are strong enough to allow the detection of individual radio pulses, for the vast majority, the pulses are buried deep within the noise of the telescope. A single timing observation therefore consists of folding the digitized telescope signal modulo the expected topocentric pulse period in a sufficiently long integration to beat down the noise. This is particularly useful because a characteristic observational property of pulsars is that the coherent summation of many individual pulses always leads to the same signature, called an "average profile," that is unique to that pulsar at that observing frequency. A fiducial point in the pulse profile, for example the pulse peak, therefore plausibly corresponds to a fixed point on the neutron star surface. Examples of two average profiles are given in Figure 1. The fiducial points chosen for the average profiles in Figure 1 are indicated by arrows. A pulse time-of-arrival (TOA) is the time at which the fiducial point of a pulse close to the mid-point of an integration arrived.

Pulsar timing consists of obtaining a sequence of TOAs, spaced typically by several weeks, over the course of many months or years. If pulsar rotation periods as observed on Earth did not change (that is, if the Earth were not accelerating with respect to the pulsar and pulsars did not lose or gain rotational energy), pulsar timing would be very simple: there would always be an integral number of neutron star rotations between any two observed TOAs, with only very small deviations resulting from measurement errors. However, life is never simple, and before the stability of pulsar rotation can be observed, one must account for a number of systematic effects. 

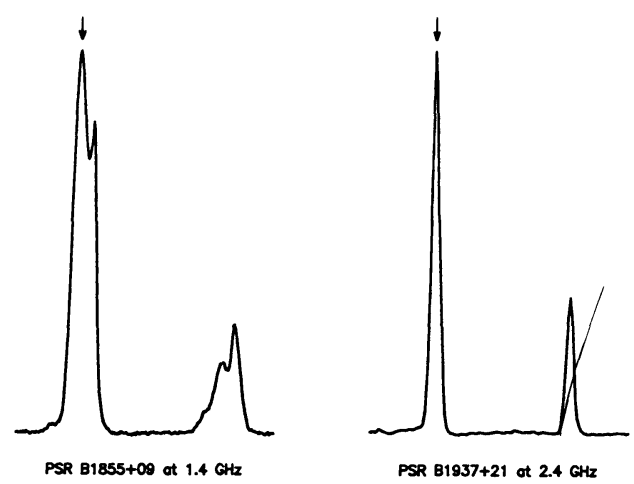

Figure 1. Average profiles for PSRs B1855+09 and B1937+21 at 1.4 and $2.4 \mathrm{GHz}$ respectively. Arrows indicate the fiducial reference points for timing.

\subsection{THE PULSAR REFERENCE FRAME}

Even if one could observe from a reference frame not accelerating with respect to the pulsar, the observed period would change since the pulsar loses energy through magnetic dipole radiation. An approximate expression for the rotation phase is obtained by Taylor expansion:

$$
\phi(t)=\phi(0)+\nu t+\frac{1}{2} \dot{\nu} t^{2}+\frac{1}{6} \ddot{\nu} t^{3}+\ldots,
$$

where $\phi(t)$ is the rotation phase in cycles at time $t, \nu \equiv 1 / P$ is the rotation frequency, and $\dot{\nu}$ and $\ddot{\nu}$ are the rotation frequency first and second derivatives. The coefficients in this expansion can be related if some physical model for the rotation is assumed. For a rotating magnetic dipole moment $\mathcal{M}$, classical electrodynamics says the total radiated power $\mathcal{P}$ is given by

$$
\mathcal{P}=\frac{2(\mathcal{M} \sin \alpha)^{2}(2 \pi \nu)^{4}}{3 c^{3}}
$$

where $\alpha$ is the angle between the spin and dipole axes. Setting the rate of loss of kinetic energy equal to the radiated power yields

$$
\dot{\nu}=\frac{8 \pi^{2}(\mathcal{M} \sin \alpha)^{2}}{3 I c^{3}} \nu^{3} \text {. }
$$

Pulsar timing typically allows the measurement of $\nu$ and $\dot{\nu}$. These in turn can be used to estimate various other quantities of interest. For example, the characteristic spin-down age $\tau$ of a pulsar can be estimated from $\tau=-\nu / 2 \dot{\nu}$, and the magnetic dipole moment $\mathcal{M}$ can be estimated via Equation 3 . It can easily be shown that $\ddot{\nu}$ from dipole radiation is negligible for most pulsars, including all millisecond pulsars. However, measured 
values of $\ddot{\nu}$ and even higher order terms often exceed the values expected from the dipole radiation model; this is generally attributed to slight instabilities in the neutron star rotation.

\subsection{THE OBSERVATORY REFERENCE FRAME}

The accurate measurement of the intrinsic spin parameters $\nu$ and $\dot{\nu}$ depends crucially on the transformation of the TOAs to the neutron star's rest frame. If this is not done correctly, the parameters may be contaminated by variable relative acceleration. A transformation to the solar system barycenter is most expedient and is sufficient for all isolated disk pulsars (those in globular clusters suffer significant accelerations in the cluster potential); the approximation that the barycenter is not accelerated with respect to the pulsar rest frame is more accurate for closer pulsars. A review of the transformation is given in Backer and Hellings (1986). We discuss the main points here.

An arrival time $t_{b}$ at the solar system barycenter is specified by

$$
t_{b}=t+\frac{\mathbf{r} \cdot \hat{\mathbf{n}}}{c}+\frac{(\mathbf{r} \cdot \hat{\mathbf{n}})^{2}-\mathbf{r}^{2}}{2 c d}-\frac{D}{f^{2}}+\Delta_{E \odot}-\Delta_{S \odot}
$$

where $t$ is the observed topocentric TOA, $\mathbf{r}$ is a vector from the barycenter to the phase center of the telescope, and $\hat{\mathbf{n}}$ is a unit vector from the barycenter to the pulsar. The terms featuring the vector $\mathbf{r}$, which incorporate the Newtonian time-of-flight of the pulsed signal between the observatory and the solar system barycenter, are referred to as the solar system "Roemer delay." The observing frequency $f$ is measured in the barycentric rest frame, and the dependence of the time delay on $f^{-2}$ is a result of the ionized interstellar plasma. The "Einstein delay" term, $\Delta_{E \odot}$, is a combination of gravitational redshift and time dilation effects due to the motion of the Earth and other objects in the solar system. The "Shapiro delay" term, $\Delta_{S \odot}$, characterizes the general relativistic curvature of spacetime near the Sun. The correct calculation of all the above therms requires an accurate planetary ephemeris such as the Jet Propulsion Laboratory's DE200 ephemeris (Standish 1982).

\subsection{BINARY PULSARS}

For pulsars having binary companions, an additional transformation is required to account for the pulsar's orbital motion. Five Keplerian parameters are necessary to describe a binary orbit in which the projected motion of one object is observed: the orbital period, the orbital eccentricity, the longitude of periastron, the epoch of periastron, and the projected semi-major axis of the pulsar orbit. In some cases, due to sufficiently small orbital 
separations, high orbital eccentricities, or favorable alignment, the Keplerian framework does not adequately describe the orbit. For these systems, "Post-Keplerian" parameters, such as time-derivatives of the Keplerian parameters, must also be included. Measurements of such parameters can be used for precise tests of relativistic gravity theories, one of the most exciting applications of pulsar timing. Details of the parametrization using various relativistic binary models are summarized by Taylor and Weisberg (1989).

\section{Pulsar Data and Precision Astrometry}

It is clear from Equation 4 that the position of the pulsar plays a key role in the timing model. Imagine a pulsar in the ecliptic at some ecliptic longitude. If the TOAs from that pulsar are not corrected for Earth's motion, delays and advances will be observed such that the differences between modelpredicted arrival times and observed arrival times, that is, the "residuals," will contain a systematic sine wave (ignoring, for the moment, the small eccentricity of the Earth's orbit) of period $1 \mathrm{yr}$ with a phase that depends on the longitude. The sine wave amplitude will be about $500 \mathrm{~s}$ (corresponding to the Earth-Sun distance), much larger than all known pulse periods. By fitting out this signal, an accurate ecliptic longitude is obtained. If the pulsar is not in the ecliptic, the sine wave amplitude is reduced, and the fit also determines the ecliptic latitude. Small amplitude 1-yr sine waves in residuals can be used to fine tune the fit position.

This measurement can be done with unprecedented precision: if the input pulsar position is wrong by $\sim 1^{\prime \prime}$, the annual sinusoid has amplitude $\sim 1 \mathrm{~ms}$, so for pulsars with TOA uncertainties of a few ms, the position can be known ideally to within a few arcseconds after only one year, with the uncertainty decreasing as the square root of the length of the data span. For millisecond pulsars, typical timing uncertainties are three orders of magnitude smaller, so far greater accuracy is easily achieved. For example, after $9 \mathrm{yr}$ of timing PSR B1937+21 with individual TOA uncertainties of $\sim 0.2 \mu \mathrm{s}$, the position uncertainty is around $50 \mu \mathrm{arcsec}$ in the reference frame defined by the DE200 planetary ephemeris.

If the pulsar has a substantial proper motion, the residuals exhibit a sine wave of linearly increasing amplitude. At a distance of $1 \mathrm{kpc}$, a pulsar moving with typical transverse velocity $200 \mathrm{~km} \mathrm{~s}^{-1}$ has proper motion of 40 mas $\mathrm{yr}^{-1}$. Thus, it is quite difficult to detect proper motions of slow pulsars from timing measurements, however for millisecond pulsars, the measurement is straightforward. Pulsar proper motions are also measurable using interferometric techniques (e.g. Harrison, Lyne and Anderson 1993).

If the pulsar is nearby, geometric parallax, which has its greatest effect when the Earth-Sun line is perpendicular to the pulsar-Sun line, results 


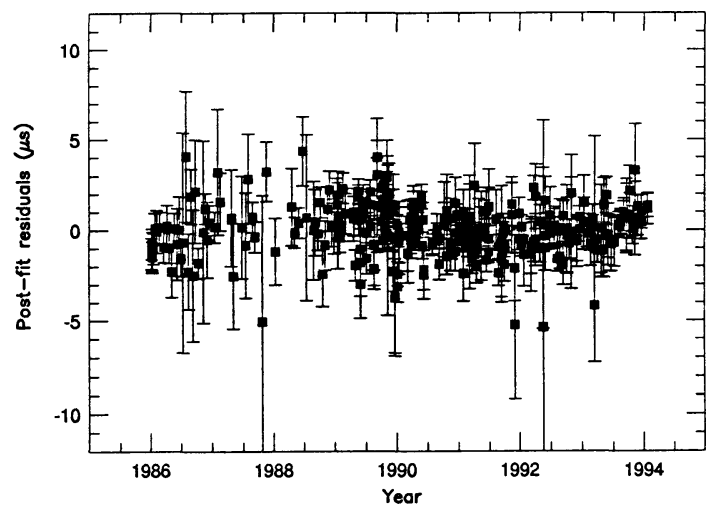

Figure 2. Post-fit residuals for PSR B1855+09 at $1.4 \mathrm{GHz}$.

in a sine wave of period $0.5 \mathrm{yr}$. (This effect is precisely described by the second Roemer term in Eq. 4). The parallax effect is tiny even by pulsar standards: for a pulsar in the plane of the ecliptic at a distance of $1 \mathrm{kpc}$, the amplitude of the parallax signal $\pi$ is only $1.2 \mu \mathrm{s}$. A measurement of $\pi$ is quite valuable however, since it allows a direct determination of the distance $d$ to a pulsar, via $\pi=1 / d$. A timing parallax has been measured for only one pulsar, PSR B1855+09, discussed below. Pulsar parallaxes can also in principle be measured directly using VLBI, although these experiments have proven to be difficult (e.g. Gwinn et al.1986).

\section{Recent Results from Arecibo}

We now describe recent results from a long-term, high precision millisecond pulsar timing project done at the $305-\mathrm{m}$ dish at Arecibo, Puerto Rico. Biweekly timing observations for two millisecond pulsars, PSRs B1855+09 and B1937+21, are described in detail in Kaspi, Taylor and Ryba (1994) (hereafter KTR94). High-precision timing of PSR B1855+09 was begun in 1986 at Arecibo at $1.4 \mathrm{GHz}$, and has yielded daily-averaged arrival time uncertainties of $\sim 1 \mu \mathrm{s}$. Data for PSR B1937+21 goes back to 1984 at 1.4 and $2.4 \mathrm{GHz}$, with daily-averaged TOA uncertainties of $\sim 0.2 \mu \mathrm{s}$. The data sets analyzed by KTR94 include arrival times obtained through the end of 1992, although here we include data through the end of 1993. All TOAs in the analysis are referred to UTC.

PSR B1855+09 is a 5.4 ms pulsar in a 12 day circular orbit with a white dwarf. The pulsar's celestial coordinates and proper motion are determined

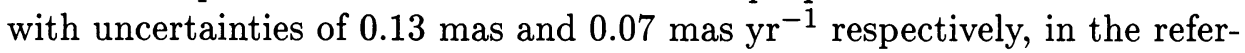
ence frame defined by the JPL DE200 planetary ephemeris. PSR B1855+09 is the only pulsar for which a significant measurement of timing parallax 
has been made. The pulsar's astrometric parameters are listed in Table 1. The residuals after removal of the best spin, astrometric and binary parameters are shown in Figure 2. The residuals are clearly dominated by random, Gaussian measurement uncertainties, which indicates that the model describes the data well. The slight hint of a cubic signal is apparent if the plot is held at a distance; this will be discussed below.

TABLE 1. Astrometric Parameters for PSRs B1855+09 and $\mathrm{B} 1937+21$

\begin{tabular}{lcc}
\hline & PSR B1855+09 & PSR B1937+21 \\
\hline$\alpha(\mathrm{J} 2000)$ & $18^{\mathrm{h}} 57^{\mathrm{m}} 36^{\mathrm{s}} .393515(4)$ & $19^{\mathrm{h}} 39^{\mathrm{m}} 38^{\mathrm{s}} .560211(2)$ \\
$\delta(\mathrm{J} 2000)$ & $+09^{\circ} 43^{\prime} 17^{\prime \prime} .32370(12)$ & $+21^{\circ} 34^{\prime} 59^{\prime \prime} .14170(4)$ \\
$\mu_{\alpha}\left(\mathrm{mas} \mathrm{yr}^{-1}\right)$ & $-2.91(4)$ & $-0.134(8)$ \\
$\mu_{\delta}\left(\mathrm{mas} \mathrm{yr}^{-1}\right)$ & $-5.48(6)$ & $-0.452(9)$ \\
$\pi(\mathrm{mas})$ & $1.0(3)$ & $<0.20$ \\
\hline
\end{tabular}

PSR B1937+21 was the first discovered and is still the fastest known millisecond pulsar, having $P=1.5 \mathrm{~ms}$. It shows no evidence for a binary companion. DE200 celestial coordinates and proper motion were measured with uncertainties of 0.05 mas and 0.01 mas $\mathrm{yr}^{-1}$. The best fit astrometric parameters are shown in Table 1, and the residuals after subtraction of the best model including astrometric and spin parameters are shown in Figure 3. There is an obvious cubic trend in the residuals that indicates our model does not completely describe the rotation of the neutron star. KTR94 address this issue in detail, and demonstrate that although there are several possible origins of the "noise," including planetary ephemeris errors, a primordial background of gravitational waves, clock errors, or some poorly understood interstellar propagation phenomenon, the most likely origin is intrinsic to the pulsar itself. The lower-level cubic of opposite sign in the residuals for PSR B1855+09 support this argument.

It should be noted that we have minimized the effect these deviations from the simple spin-down model have on the astrometric parameters for PSR B1855+09 and especially PSR B1937+21 by "whitening," that is, by including sufficiently many higher order frequency derivatives to render the residuals random when determining the astrometric parameters.

\section{Frame Ties and the Future}

The high precision astrometry available through millisecond pulsar timing is useful for tying astronomical reference frames. An interferometric po- 


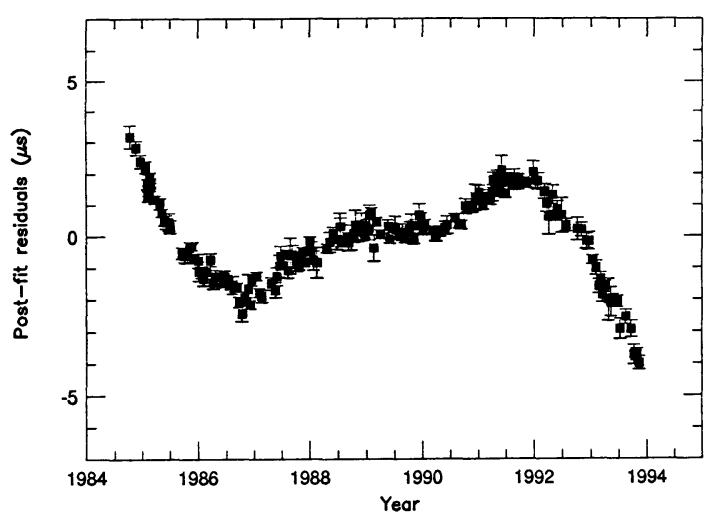

Figure 3. Post-fit residuals for PSR B1937+21 at $2.4 \mathrm{GHz}$.

sition for a pulsar together with a timing position, for example, tie the extragalactic reference frame with that defined by solar system objects. At $400 \mathrm{MHz}$, PSR B1937+21 is a $240 \mathrm{mJy}$ source so is easily observable with VLBI techniques. Indeed Bartel et al. (1990) have observed the pulsar and obtained a VLBI position suitable for a frame tie. PSR B1855+09 by contrast is only $31 \mathrm{mJy}$ at $400 \mathrm{MHz}$, and so is not well-suited to this technique.

An optical detection of a pulsar or, more likely, of the companions to binary millisecond pulsars, can in principle provide a tie with the optical reference frame. Although under $10 \%$ of the slow pulsar population are in binary systems, well over $75 \%$ of disk millisecond pulsars are in binaries, with the majority having a potentially optically observable companion. The detection of optical companions to millisecond pulsars is also interesting from an evolutionary point of view (Kulkarni et al.1991). The advent of HST and other sensitive instruments such as the Keck Telescope will be a great boon to the detection of very faint companions, as well as to improved astrometry for brighter sources.

One newly discovered source deserves notice. PSR J0437-4715, discovered by Johnston et al. (1993), is a $5.7 \mathrm{~ms}$ pulsar in a 5 day circular orbit with a white dwarf. It is a $600 \mathrm{mJy}$ source at $400 \mathrm{MHz}$, and is also one of the closest pulsars, with an estimated distance of only $140 \mathrm{pc}$. The source will be easily detectable by VLBI, and furthermore, the pulsar's companion has already been detected optically by Danziger et al. (1993). After only two years of timing, the timing position and proper motion have been measured

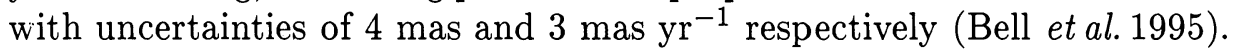

The number of millisecond pulsar discoveries has sky-rocketed in recent years (see Fig. 4) owing principally to large-scale search efforts. There is every reason to believe that millisecond pulsar timing will be a booming industry in the years to come, and that precise positions and proper mo- 


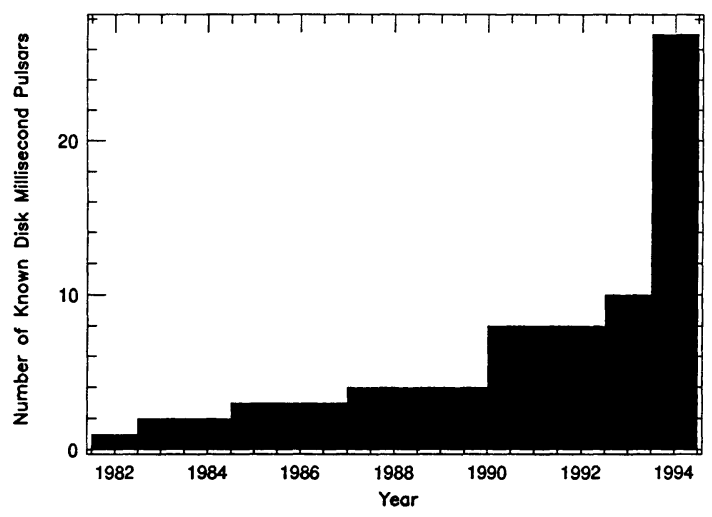

Figure 4. Number of known disk millisecond pulsars versus year.

tions will be available in the next decade for a large number of sources. Furthermore, as more millisecond pulsars are discovered, it is becoming clear that they are distributed isotropically on the sky; the possibility of a complete tie of optical, extragalactic, and dynamical reference frames from observations of millisecond pulsars may soon be within reach.

\section{Acknowledgements}

I am indebted to Joe Taylor for his foresight and advice. I also thank F. Camilo, D. Nice, and P. Ray for discussions. Financial support was provided by a Princeton University Higgins Instructorship, and by a Hubble Fellowship through NASA grant number HF-1061.01-94A from the Space Telescope Science Institute, which is operated by the Association of Universities for Research in Astronomy, Inc., under NASA contract NAS5-26555.

\section{References}

Backer, D. C. \& Hellings, R. W., 1986. Ann. Rev. Astr. Ap., 24, 537.

Bartel, N., Cappalo, R., Whitney, A., Chandler, J., Ratner, M., Shapiro, I. \& Tang, G., 1990. In: Impact of Pulsar Timing on Relativity and Cosmology, X1, ed. Backer, D., Center for Particle Astrophysics, Berkeley.

Bell, J., Bailes, M., Manchester, R. N., Weisberg, J. \& Lyne, A. G., 1995. in preparation.

Danziger, I. J., Baade, D. \& Della Valle, M., 1993. Astr. Astrophys., 276, 382.

Gwinn, C. R., Taylor, J. H., Weisberg, J. M. \& Rawley, L. A., 1986. Astron. J., $91,338$.

Harrison, P. A., Lyne, A. G. \& Anderson, B., 1993. Mon. Not. R. astr. Soc., in press.

Johnston, S., Lorimer, D. R., Harrison, P. A., Bailes, M., Lyne, A. G., Bell, J. F., Kaspi, V. M., Manchester, R. N., D'Amico, N., Nicastro, L. \& Jin, S., 1993. Nature, 361, 613.

Kaspi, V. M., Taylor, J. H. \& Ryba, M., 1994. Astrophys. J., 428, 713.

Kulkarni, S. R., Djorgovski, S. \& Klemola, A. R., 1991. Astrophys. J., 367, 221.

Standish, E. M., 1982. Astr. Astrophys., 114, 297.

Taylor, J. H. \& Weisberg, J. M., 1989. Astrophys. J., 345, 434. 